\title{
Quantifying Users' Interconnectedness in Online Social Networks - An Indispensible Step for Economic Valuation
}

\author{
by
}

Martin Gneiser, J ulia Heidemann, Mathias Klier, Andrea Landherr, Florian Probst

in: M. Nelson, M. Shaw, T. Strader, eds., Value Creation in E-Business

Management - 15th Americas Conference on Information Systems, AMCIS, SIGeBIZ track, San Francisco, California, August 2009, p. 246-258 


\title{
Quantifying Users' Interconnectedness in Online Social Networks - An Indispensible Step for Economic Valuation
}

\author{
Martin Gneiser \\ FIM Research Center \\ Finance \& Information Management, \\ University of Augsburg, Germany \\ martin.gneiser@wiwi.uni-augsburg.de \\ Mathias Klier \\ Department of Information Systems, \\ University of Innsbruck, Austria \\ mathias.klier@uibk.ac.at
}

\author{
Julia Heidemann \\ FIM Research Center \\ Finance \& Information Management, \\ University of Augsburg, Germany \\ julia.heidemann@wiwi.uni-augsburg.de \\ Andrea Landherr \\ FIM Research Center \\ Finance \& Information Management, \\ University of Augsburg, Germany \\ andrea.landherr@gmx.de
}

\author{
Florian Probst \\ FIM Research Center \\ Finance \& Information Management, \\ University of Augsburg, Germany \\ florian.probst@eliteakademie.de
}

\begin{abstract}
Online social networks have been gaining increasing economic importance in light of the rising number of their users. Numerous recent acquisitions priced at enormous amounts have illustrated this development and revealed the need for adequate business valuation models. The value of an online social network is largely determined by the value of its users, the relationships between these users, and the resulting network effects. Therefore, the interconnectedness of a user within the network has to be considered explicitly to get a reasonable estimate for the economic value. Established standard business valuation models, however, do not sufficiently take these aspects into account. Thus, we propose a measure based on the PageRank-algorithm to quantify users' interconnectedness in an online social network. This is a first but indispensible step towards an adequate economic valuation of online social networks.
\end{abstract}

\section{Keywords}

Online social networks, economic valuation, centrality measures, PageRank.

\section{INTRODUCTION}

Thanks to a variety of online social applications, including blogs, user-generated content sites like YouTube.com and countless online communities across the World Wide Web (WWW), people are connecting and communicating more and more online with one other (Bernoff and $\mathrm{Li}, 2008$ ). Along with these changes, formerly passive information users are becoming actors, which create the content of the WWW themselves. In this context, online social networks (OSN) are currently of particular interest. Therefore, networking platforms such as MySpace.com and Facebook.com have spurred enormous attention among researchers and practitioners. The active use of OSN enjoys great popularity both in private and corporate context. According to a recently published study conducted by the European Interactive Advertising Association (EIAA), 42\% of all European Internet users participate in OSN (EIAA, 2008). Moreover, Emarketer.com states that 37\% of US adult and $70 \%$ of US teenage Internet users used OSN every month in 2007 (Williamson, 2007). With the growing number of users, this technical and social phenomenon generates an increasingly important economic impact. Thus, media and IT companies have been acquiring OSN for considerable amounts to adapt their business models to the new environmental conditions and to reorganize their companies for the future. In 2005, for example, the media company News Corp. acquired the OSN MySpace.com for US\$ 580 million (BBC, 2005). Two years later, Microsoft paid US\$ 240 million 
for a 1.6\% minority interest in the OSN Facebook.com (Hofmann and Knahl, 2008). The extrapolated value of this company thus amounts to staggering US\$ 15 billion. However, the enormous purchase prices for OSN are also being considered critical and experts compare the situation with the speculative dotcom bubble before the turn of the millennium. Martin Sorrell for instance, CEO of the WWP Group is seriously questioning the valuation of Facebook.com at US\$ 15 billion (Lambrecht, 2008).

What makes the economic valuation of OSN difficult is that the value of OSN is largely determined by the value of its users, the relationships between these users, and the resulting network effects. For instance, with a growing number of individual contacts, the attractiveness of an OSN increases for every single user, i.e. a well-connected user might use an OSN more actively, and attracting new contacts within and beyond the network. Furthermore, the loyalty of a user strongly depends on the integration into the OSN, since every additional contact raises the barrier to leave the network (Algesheimer and von Wangenheim, 2006). Consequently, the interconnectedness of each user in the OSN has to be considered explicitly to get a reasonable estimate for the company value. Currently, established standard business valuation models do not sufficiently consider this aspect. Thus, the important question of how OSN can be valued using well-founded valuation methods while considering the interconnectedness of its users has not been answered yet. Therefore, the focus of this paper is quantifying users' interconnectedness in OSN which is a first but indispensible step towards an adequate economic valuation of OSN.

The paper is structured as follows: First, we define OSN, derive requirements which an adequate measure for quantifying a user's interconnectedness in OSN has to fulfill, and briefly review existing centrality measures. Then, we propose a new quantitative measure based on the PageRank-algorithm, before we illustrate the application of the measure by an example. The last section summarizes the results and points out areas for further research.

\section{BACKGROUND}

\section{Definition of Online Social Networks}

Aroused by the web 2.0 boom, OSN have evolved into a new, mostly free of cost mass medium where users ${ }^{1}$ present themselves to a wide public. They voluntarily reveal parts of their privacy and establish or maintain connections with other users. Besides the exponential growth of OSN, the way they are perceived has changed over the last years. OSN are not simply forums in which individuals congregate. They rather "create substantial value for the individuals who participate in them, the organizations that sponsor them, and the larger society in multiple ways” (Agarwal, Gupta and Kraut, 2008). In the following we define - according to Boyd and Ellison (2007) - OSN in particular as "web-based services that allow individuals to (1) construct a public or semi-public profile within a bounded system, (2) articulate a list of other users with whom they share a connection, and (3) view and traverse their list of connections and those made by others within the system". Thereby, the aspect of networking, i.e. establishing and maintaining relationships between users, plays a decisive role. Currently, there are a lot of OSN both for business (e.g. Doostang.com, LinkedIn.com) and private purposes (e.g. Facebook.com, MySpace.com) aiming at different target groups. While the culture that emerges around OSN varies, the maintenance of individual contacts and most of the key technological features are fairly consistent (Boyd and Ellison, 2007). Furthermore, the community idea is actively lived over forum and group functions and network structures are observable (Xu, Zhang, Xue and Yeo, 2008).

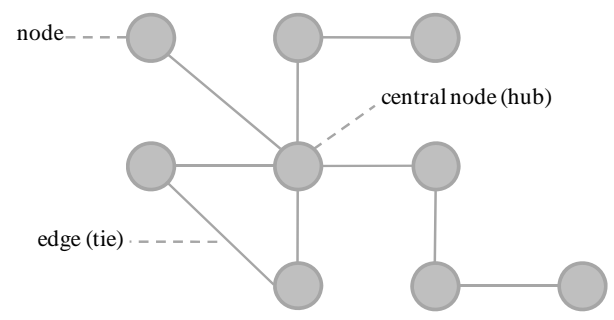

Figure 1. Visualization of Network Structure

In social network analysis this network structure is perceived as a set of actors, which are represented by nodes, and a set of edges (ties) linking pairs of nodes (Adamic and Adar, 2003; Bampo, Ewing, Mather, Stewart and Wallace, 2008; Wasserman

\footnotetext{
${ }^{1}$ The terms customer and user are used synonymously.
} 
and Faust, 1994). The edges represent connections between actors and describe social interactions or relationships. The nodes and edges are usually presented by a graph (Hanneman and Riddle, 2005), as shown in Figure 1.

\section{Economic Valuation of Online Social Networks}

A plethora of articles and books on the valuation of companies in general has been published (Brealey and Myers, 2008; Koller, Goedhart and Wessels, 2005). However, according to the predominant view in literature standard business valuation approaches are very restricted in their ability to value young, fast growing companies in a dynamic environment, such as Internet companies (see e.g. Gollotto and Kim, 2003). For OSN, the economic valuation is even more difficult, since users, relationships between users, and the resulting network effects represent a major part of the company value. Hence, the value of each user per se and the importance of a user within the OSN have to be considered explicitly to get a reasonable estimate for the company value. Established standard business valuation models do not comprehensively take these aspects into account yet. However, in recent years new approaches have been developed, which consider the value of customers as the most important factor for a company’s valuation (see e.g. Bauer and Hammerschmidt, 2005; Gupta, Lehmann and Stuart, 2004). Nevertheless, these valuation approaches have a major drawback concerning the application to OSN: network effects resulting from relationships between users are ignored. This is crucial, since a user, providing no direct financial returns to a company, might have - if considered isolated - a low (or even no) value. Yet, he or she might affect many other users by interacting with them and hence, for instance, motivate them to stay members of the network (Kiss and Bichler, 2008)

In general, network effects and in particular the importance of relationships between users within a social network have been an extensively and well-researched field in (social) network analysis. Network effects are thereby characterized by dependencies between the increasing utility that a user derives from consumption of a good and the growing number of other agents consuming this good (Katz and Shapiro, 1985, 1994; Shapiro and Varian, 1998). Bass (1969) thereby differentiates between innovators, i.e. agents adopting an innovation independently of others in a social system, and adopters. Individual utility models of the diffusion of innovations state that people adopt new technologies if benefits from adoption and use exceed the costs (Rogers, 2003). Besides the social component that influences this utility - e.g. number of other users of an innovation - normative models play a decisive role (Kraut, Rice, Cool and Fish, 1998). Especially adopters are influenced by the pressures of the social system that increase with the number of previous adopters (Bass, 1969). An individual's social influence in the adoption process is likely to be highly dependent on the position in a social network (Kraut et al. 1998, Rice and Aydin 1991).

The spread of a certain behavior or innovation in a social network, however, is not always invoked by a group of wellconnected hubs (Watts, 2007). Only if a critical mass of users is exceeded (Arthur, 1989; Morris and Ogan, 1996), new users are attracted and a stronger interconnectedness of the users leads to so-called lock-in-effects (Farrell and Shapiro, 1989; Shapiro and Varian, 1998). A central position of an individual nevertheless positively affects his or her influence in and value for a network. The OSN XING.com, for instance, reports that well-connected users have (due to network effects) a higher retention rate (i.e. they are less inclined to leave the network), attract new users to a greater extent and lead to a higher activity among users (XING, 2006). So, the number of users and their interconnectedness are crucial issues when valuating OSN (Algesheimer and von Wangenheim, 2006).

Besides these general analyses, particularly social network analysts have focused on describing networks of relations for instance by tracing the flow of information through them and discovering the effects of these relations and networks on people and organizations (Wasserman and Faust, 1994; Berkowitz, 1982). Granovetter (1973), for example, analyzed the strength of relationships (ties) between people in detail, classifying them to be strong, weak or absent. He remarks, that strong ties have greater motivation to be of assistance and are typically more easily available. However, weak ties provide people with access to information and resources beyond those available in their own social circle (Granovetter, 1973; Granovetter, 1983) and bridge cliques of strong ties (Constant, Sproull and Kiesler, 1996). Hence, OSN allow users to draw on resources from other users of the network and to leverage connections from multiple social and geographically dispersed contexts (Haythornthwaite, 2002). Furthermore, Watts and Strogatz (1998) found that almost all social networks are scalefree, i.e. they have a structure with many nodes with only few connections and some hubs creating short cuts between nodes which otherwise would be far away form each other. Thus, even though there might be gaps between individuals within large OSN, i.e. there are no direct links among all participants, well-connected users tie together sub-networks. So, the different roles and characteristics of users concerning their integration have to be taken into account adequately when valuating OSN.

A number of experiments, constructing paths through social networks to distant target individuals (e.g. Dodds, Muhamad, Watts, 2003; Garfield, 1979; Korte and Milgram, 1970) and current studies (e.g. Leskovec and Horvitz, 2008) confirm the socalled "small world" effect regarding modern networks such as the Internet and OSN. This phenomenon goes back to Stanley Milgram (1967), who provided first empirical support for the notion that everyone is just a few steps apart in the global social 
network. Therefore, it is not sufficient to consider only parts of the OSN (sub-networks) when investigating a user's integration in the OSN. In fact, the complete network and all users and relationships have to be taken into account when analyzing the interconnectedness of the users for valuation purposes.

It is remarkable that despite the extensive research in network theory - to the best of our knowledge - no approach for the economic valuation of OSN, which adequately takes into account the users' interconnectedness in the network has been developed so far. Therefore, this paper focuses on quantifying users' interconnectedness in OSN, which is a first but indispensible step towards an appropriate economic valuation of OSN. In the following, we derive three requirements an adequate measure has to fulfill. They will be used to evaluate common centrality measures regarding their ability to quantify a user's interconnectedness for the economic valuation of OSN. They will also serve as guidelines when proposing an adapted quantitative measure.

As argued above, the number of direct contacts (“neighbors”) plays an important role for the interconnectedness of a user:

R.1 [Consideration of direct contacts] A user's direct contacts have to be taken into account adequately when quantifying his or her interconnectedness.

With regard to OSN, a connection to a user with many contacts might be more valuable than to a user with only one or no further contact (Kiss and Bichler, 2008). Hence, the interconnectedness of a user's direct contacts (i.e. his or her indirect contacts) has to be considered when quantifying the user's interconnectedness:

R.2 [Consideration of indirect contacts] The interconnectedness of a user's contacts (indirect contacts and their network) has to be considered adequately when quantifying his or her interconnectedness.

To enable the application of the measure in real-world scenarios we additionally state one more requirement:

R.3 [Feasibility] The measure should be based on determinable input data, its computational complexity should be manageable, and from an economic point of view it is required that the measurement can be accomplished at a high level of automation.

\section{METHOD}

\section{Discussion of Common Centrality Measures}

The quantification of interconnectedness in networks in general has attracted attention not only in social network analysis but also in many other fields (e.g. biology, physics). In the context of social networks, the most common so-called centrality measures are presented in Freeman's article "Centrality in Social Networks" (Freeman, 1979). These measures can be used to quantify the importance of a certain node within a network. In the following, we provide the definition of each of the three measures and analyze their ability to quantify a user's interconnectedness for the economic valuation of OSN on the basis of the requirements R.1-R.3.

\section{Degree Centrality}

The basic idea of degree centrality is that a node with many direct connections to other nodes is central to the network (Sparrowe, Liden, Wayne and Kraimer, 2001). Thus, this measure is based upon the number of a node's direct contacts. For a node $i \in\{1, \ldots, n\}$, degree centrality is defined as

$$
C_{D}(i)=\sum_{\substack{j=1 \\ j \neq i}}^{n} a_{i j}
$$

where $n \in \mathrm{IN}$ is the number of nodes in the network and $a_{i j} \in\{0,1\}$ is an element of the adjacency matrix which is 1 if and only if there exists an edge between the nodes $i$ and $j$ (otherwise it is 0) (Freeman, 1979). Degree centrality considers direct contacts (R.1) and is easy to compute from network data (R.3). However, indirect contacts are not considered at all. Therefore R.2 is not fulfilled.

\section{Betweenness Centrality}

Betweenness centrality quantifies the ability of a node to reach other nodes in the network. Freeman (1979) defines it as the frequency with which a node falls between all unordered pairs of other nodes on the shortest paths connecting them. For a node $i \in\{1, \ldots, n\}$ within a connected network, betweenness centrality is defined as 


$$
C_{B}(i)=\sum_{\substack{j=1 \\ j \neq i}}^{n} \sum_{\substack{k=j+1 \\ k \neq i}}^{n} \frac{g_{j k}(i)}{g_{j k}},
$$

where $g_{j k} \in \mathrm{IN}$ is the number of shortest paths linking $j$ and $k$ and $g_{j k}(i) \in \mathrm{IN}$ is the number of these paths containing node $i$. Betweenness centrality does not take into account direct or indirect contacts adequately for the economic valuation of OSN as all connections between users are important in OSN (not only the shortest paths). In figure 2, for example, the values for betweenness centrality are 0 for node 1 and 2, although both nodes have direct and indirect contacts. Furthermore, the values are the same for node 1 and 2, although node 2 has more direct contacts. In conclusion, neither R.1 nor R.2 is completely fulfilled. As there exist adequate algorithms to overcome computational performance problems R.3 is fulfilled.

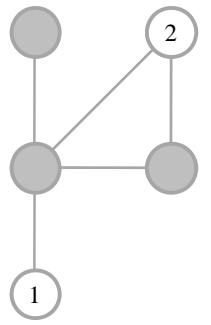

$$
\begin{aligned}
& C_{B}(1)=0 \\
& C_{B}(2)=0
\end{aligned}
$$

Figure 2. Betweenness Centrality (Example)

\section{Closeness Centrality}

The concept of closeness centrality considers a node as central, if it is at short distance to all other nodes in the network. For a node $i \in\{1, \ldots, n\}$ closeness centrality is defined as the reciprocal value of the sum of shortest distances from a node to all other nodes in the network and can be denoted as

$$
C_{C}(i)=\frac{1}{\sum_{\substack{j=1 \\ i \neq j}}^{n} d(i, j)},
$$

where $d(i, j) \in \mathrm{IN}$ is the minimum length of any path connecting $i$ and $j$ (Freeman, 1979). It accounts for direct contacts (R.1). However, indirect contacts are not considered adequately (R.2) as the interconnectedness of a user's direct contacts is not taken into account consistently. In figure 3, for instance, closeness centrality returns the same values for node 1 in network a) and $b$ ), although the interconnectedness of the contacts of node 1 in network b) is much higher (additional edges). Therefore R.2 is not completely fulfilled. Although closeness centrality is relatively difficult to calculate, adequate algorithms for computing distances in a network exist. So R.3 is fulfilled.

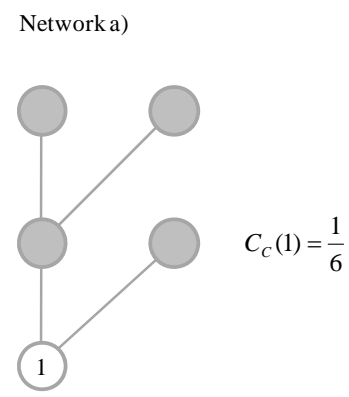

Networkb)

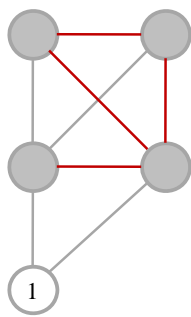

$C_{C}(1)=\frac{1}{6}$

Figure 3. Closeness Centrality (Example) 


\section{A New, PageRank Based Centrality Measure for Online Social Networks}

None of the centrality measures discussed so far fulfills all requirements (R.1-R.3) for the economic valuation of OSN. Particularly indirect contacts are not considered adequately (R.2). But with regard to OSN, a connection to a node with high interconnectedness (i.e. with many direct and indirect contacts) is - as already discussed - more valuable than a connection to a sparsely connected node. However, measures accounting for this fact have been already developed in the context of Web search engines: Brin and Page (1998), the founders of the Google Internet search engine, developed the popular PageRankalgorithm to rank the importance of Web pages - a problem very similar to the quantification of a user's interconnectedness in OSN. In the following we discuss PageRank as a possible centrality measure and adapt it to the context of OSN.

PageRank is based on a graph where Web pages are nodes and (directed) edges represent the links between them. PageRank uses the link structure as an indicator of an individual page's value relative to other pages by interpreting a link from page A to page B as a vote by page A for page B. At the same time, it analyzes the page that casts the vote. Brin and Page (1998) define the PageRank for a page $i$ as

$$
P R(i)=c \cdot \sum_{j \in B_{i}} \frac{P R(j)}{N_{j}}+c \cdot E(i),
$$

such that $c$ is maximized and $\|P R\|_{1}=1\left(\|P R\|_{1}\right.$ denotes the $\mathrm{L}_{1}$ norm of $\left.P R\right)$. In formula (1), $N_{j}$ is the number of outgoing links from page $j . B_{i}$ denotes the set of pages pointing to $i$ and $E(i)$ corresponds to an additional source of rank over the Web pages. The factor $c$ is used for normalization, so that the total rank of all Web pages is constant (Brin and Page, 1998). The first part of formula (1) can be interpreted as the behavior of a "random surfer" clicking on successive links at random. It corresponds to the standing probability distribution of a random walk on the graph of the Web. However, if a real Web surfer gets into a loop of pages, he is unlikely to continue there forever. Instead, the surfer will jump to an arbitrary page. This behavior is modeled by the second part of formula (1). Methodically PageRank is a variant of eigenvector centrality. The idea of eigenvector centrality is that the centrality of node $i$ is a function of the centralities of all nodes connected to $i$. Therefore, in the first part of formula (1) node $i$ inherits a proportion of rank from all nodes pointing to it, i.e. all nodes connected to $i$ by ingoing edges. To calculate the proportion which node $i$ inherits from each node $j$ in $B_{i}$, node $j$ 's rank is divided by the number of $j$ 's outgoing edges. Hence, node $j$ contributes equally to the ranks of all pages it points to. In the second part of formula (1), $E(i)$ represents an additional source of rank for node $i$ and can be used to adjust page ranks individually.

Due to these characteristics PageRank is appropriate regarding our requirements for the quantification of interconnectedness in OSN. However, a general difference between interconnectedness in the WWW and in OSN exists: While relationships in the WWW are directed (ingoing and outgoing edges), relationships in OSN are usually seen as symmetric (undirected). Therefore, the PageRank-formula has to be adapted: $B_{i}$ (set of nodes connected to $i$ by ingoing edges) is substituted by $F_{i}$ (set of nodes connected to $i$ ). Furthermore, to assure that requirement R.2 is fulfilled and to avoid a decrease of the interconnectedness score inherited from node $j$ as the number of $j$ 's contacts grows, the dominator $N_{j}$ needs to be removed. The adapted formula is denoted as

$$
S(i)=c \cdot \sum_{j \in F_{i}} S(j)+c \cdot E(i)
$$

such that $c$ is maximized and $\|S\|_{1}=1 . F_{i}$ represents the set of user $i$ 's direct contacts. The first part of formula (2) shows the interconnectedness score, which a node $i$ inherits from its contacts. Due to the summation over $F_{i}$, all direct contacts contribute to $S(i)$. Furthermore, the (adapted) PageRank $S(i)$ of a node is calculated recursively. Thus, a node ceteris paribus inherits a higher interconnectedness score from a well-connected node than from a sparsely connected one. Therefore, direct (R.1) and indirect contacts (R.2) are considered consistently and adequately. As in the original PageRank-formula, E(i) represents an additional source of rank and can be used to account for further individual parameters (besides direct and indirect contacts) influencing a node's interconnectedness (e.g. group memberships, etc.) in an OSN. However, E(i) can be set equal to 0 if an additional source of rank is absent or ignored. As the computation of the adapted PageRank can be traced back to the problem of finding an eigenvector (cp. following example), the computational complexity can be reduced to $O\left(n^{2}\right)$ which is feasible with today's computing power (R.3).

\section{ILLUSTRATION OF THE NEW CENTRALITY MEASURE AND DISCUSSION OF THE RESULTS}

For the purpose of illustration, the adapted PageRank is calculated for an exemplary OSN, consisting of nine nodes $(i=1, \ldots, 9)$. The graph and the corresponding adjacency matrix $A$ are shown in figure 4 : 

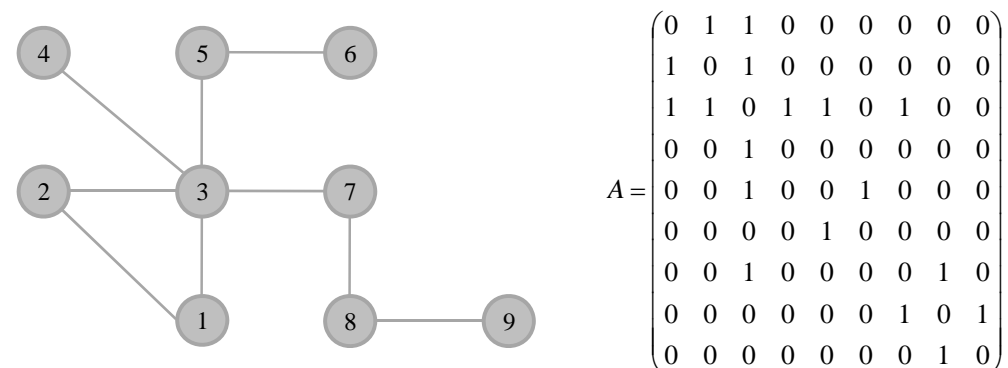

Figure 4. Illustration of the Exemplary OSN

To calculate $S(i)$ for all nodes $i$, formula (2) can be written as

$$
S=c \cdot A \cdot S+C \cdot E,
$$

where $S$ is the vector of the adapted PageRank $S(i)$ and $A$ the adjacency matrix with elements $a_{i j}=1$ if there exists an edge between the nodes $i$ and $j$ (otherwise $a_{i j}=0$ ). $E$ represents the vector assembling the additional source of rank $E(i)$ on the nodes' interconnectedness.

Solving equation (3) leads ${ }^{2}$ to the following vector of adapted PageRank values ${ }^{3}$ :

$$
S^{T}=(0.40307,0.40307,0.63691,0.24685,0.29048,0.11258,0.29985,0.13676,0.05300)
$$

Table 1 summarizes the results, illustrating each node i’s centrality position in the network in descending order.

\begin{tabular}{|c|c|c|}
\hline $\begin{array}{c}\text { Node i's } \\
\text { centrality position }\end{array}$ & Node i & $\begin{array}{c}\text { Adapted } \\
\text { PageRank S(i) }\end{array}$ \\
\hline 1 & 3 & 0.63691 \\
\hline 2 & 1 & 0.40307 \\
\hline & 2 & 0.40307 \\
\hline 4 & 7 & 0.29985 \\
\hline 5 & 5 & 0.29048 \\
\hline 6 & 4 & 0.24685 \\
\hline 7 & 8 & 0.13676 \\
\hline 8 & 6 & 0.11258 \\
\hline 9 & 9 & 0.05300 \\
\hline
\end{tabular}

Table 1. Values of the New, PageRank based Centrality Measure (Example)

For the purpose of illustration of R.2, nodes 4, 6, and 9 are considered in detail. All of these nodes have one direct contact (see figure 4). Nevertheless, their interconnectedness score $S(i)$ differs. This effect is due to the influence of indirect contacts, i.e. interconnectedness of a user's direct contacts. For example, node 4 is directly connected with node 3 , which is the node with the highest interconnectedness in the network. Node 6, in comparison, is connected to node 5, which is less connected. Hence, the interconnectedness score of node 6 is lower. Accordingly, node 9 has the lowest interconnectedness score in this example, since its only direct contact - node 8 - has a lower interconnectedness score than the other nodes' direct contacts.

Table 2 summarizes the evaluation of centrality measures regarding the requirements R.1-R.3. The table shows that the new, PageRank based centrality measure is the only one which fulfills all requirements sufficiently.

\footnotetext{
${ }^{2}$ For details regarding the general procedure for solving such kind of equations see e.g. Brin and Page (1998).

${ }^{3} E$ is considered to be the zero vector in this example.
} 


\begin{tabular}{|l|c|c|c|c|}
\hline Requirement & $\begin{array}{c}\text { Degree } \\
\text { centrality }\end{array}$ & $\begin{array}{c}\text { Betweenness } \\
\text { centrality }\end{array}$ & $\begin{array}{c}\text { Closeness } \\
\text { centrality }\end{array}$ & $\begin{array}{c}\text { Adapted } \\
\text { PageRank }\end{array}$ \\
\hline R.1 Consideration of direct contacts & yes & no & yes & yes \\
\hline R.2 Consideration of indirect contacts & no & no & no & yes \\
\hline R.3 Feasibility & yes & yes & yes & yes \\
\hline
\end{tabular}

Table 2. Evaluation of Centrality Measures Regarding the Requirements R.1-R.3

\section{SUMMARY}

The increasing economic relevance of OSN and numerous acquisitions priced at enormous amounts revealed the need for adequate valuation models. However, standard businesses valuation approaches are restricted in their ability to value OSN. A major drawback concerning the application to OSN is that the network effects resulting from relationships between users are not taken into account. Thus, we focused on a first but indispensible step towards an adequate economic valuation of OSN: the quantification of the users' interconnectedness in OSN. For this purpose a set of requirements was derived. As neither of the common centrality measures we discussed fulfills all these requirements, we propose a new centrality measure based on PageRank, an algorithm accounting for the importance of Web pages in the context of Web search engines. An exemplary network was used to illustrate the application of the adapted PageRank-algorithm. Although the findings are promising, an evaluation of the adopted PageRank-algorithm using empirical datasets is essential. Hence, we are currently working on an evaluation using publicly available social network datasets as well as empirical data provided by a German OSN. However, the integration of the users into the network is only one aspect regarding the economic valuation of OSN. Additional aspects, such as the users' activity (e.g. frequency of login, forum and chat contribution) should be considered, too. Therefore, the adapted PageRank-algorithm needs to be further enhanced. Moreover, it is important to integrate the measure for users' interconnectedness into economic valuation approaches, e.g. based on customer lifetime values.

\section{REFERENCES}

1. Adamic, L. A. and Adar, E. (2003) Friends and neighbors on the Web, Social Networks, 25, 3, 211-230.

2. Agarwal, R., Gupta, A. K. and Kraut, R. (2008) The Interplay Between Digital and Social Networks (Editorial Overview), Information Systems Research, 19, 3, 243-252.

3. Algesheimer, R. and von Wangenheim, F. (2006) A Network Based Approach to Customer Equity Management, Journal of Relationship Marketing, 5, 1, 39-57.

4. Arthur, W. B. (1989) Competing technologies, increasing returns, and lock-in by historical events, The Economic Journal, 99, 394, 116-131.

5. Bampo, M., Ewing, M. T., Mather, D. R., Stewart, D. and Wallace, M. (2008) The Effects of the Social Structure of Digital Networks on Viral Marketing Performance, Information Systems Research, 19, 3, 273-290.

6. Bass, F. (1969) A new product growth model for consumer durables, Management Science, 15, 5, $215-227$.

7. Bauer, H. H. and Hammerschmidt, M. (2005) Customer-based corporate valuation - Integrating the concepts of customer equity and shareholder value, Management Decision, 43, 3, 331-348.

8. BBC (2005) News Corp in \$580m Internet buy, http://news.bbc.co.uk/2/hi/business/4695495.stm, 2009-02-19.

9. Berkowitz, S. D. (1982) An introduction to structural analysis: The network approach to social research, Butterworth, Toronto.

10. Bernoff, J. and Li, C. (2008) Harnessing the Power of the Oh-So-Social Web, MIT Sloan Management Review, 49, 3, 3642.

11. Boyd, D. M. and Ellison, N. B. (2007) Social Network Sites: Definition, History, and Scholarship, Journal of ComputerMediated Communication, 13, 1, 210-230.

12. Brealey, R. A. and Myers, S. C. (2008) Principles of Corporate Finance, McGraw-Hill, Inc., New York. 
13. Brin, S. and Page, L. (1998) The anatomy of a large-scale hypertextual Web search engine, Computer Networks and ISDN Systems, 30, 1-7, 107-117.

14. Constant, D., Sproull, L. and Kiesler, S. (1996) The Kindness of Strangers: The Usefulness of Electronic Weak Ties for Technical Advice, Organization Science, 7, 2, 119-135.

15. Dodds, P. S., Muhamad, R. and Watts, D. J. (2003) An Experimental Study of Search in Global Social Networks, Science, 301, 5634, 827-829.

16. EIAA (2008) Shifting Traditions: Internet Rivalling TV In Media Consumption Stakes, http://www.eiaa.net/news/eiaa-articles-details.asp?lang=1\&id=154, 2009-02-12.

17. Farrell, J. and Shapiro, C. (1989) Optimal Contracts with Lock-in, American Economics Review, 79, 1, 51-68.

18. Freeman, L. C. (1979) Centrality in social networks: Conceptual clarification, Social Networks, 1, 3, $215-239$.

19. Garfield, E. (1979) It's a small world after all, Current Contents, 43, 5-10.

20. Gollotto, J. C. and Kim, S. (2003) Market valuation of Dot Com companies; R\&D versus hype, Managerial Finance, 29, $11,61-72$.

21. Granovetter, M. S. (1973) The Strength of Weak Ties, American Journal of Sociology, 78, 6, 1360-1380.

22. Granovetter, M. S. (1983) The strength of weak ties: A network theory revisited, Sociological Theory, 1, 1, $201-233$.

23. Gupta, S., Lehmann, D. R. and Stuart, J. A. (2004) Valuing Customers, Journal of Marketing Research, 41, 1, 7-18.

24. Hanneman, R. A. and Riddle, M. (2005) Introduction to Social Network Methods, http://www.faculty.ucr.edu/ hanneman/nettext/, 2009-02-12.

25. Haythornthwaite, C. (2002) Building social networks via computer networks: Creating and sustaining distributed learning communities, in K. Ann Renninger and Wesley Shumar (Eds.) Building Virtual Communities: Learning and Change in Cyberspace, Cambridge University Press, Cambridge, 159-190.

26. Hofmann, H. D. and Knahl, M. H. (2008) The Web 2.0 Demystified - Six Theses on a Misinterpreted Concept, in Proceedings of the Seventh International Network Conference, July 8-10, Plymouth, UK, 245-259.

27. Katz, M. L. and Shapiro, C. (1985) Network Externalities, Competition, and Compatibility, The American Economic Review, 75, 3, 424-440.

28. Katz, M. L. and Shapiro, C. (1994) Systems Competition and Network Effects, Journal of Economic Perspectives, 8, 2, 93-115.

29. Kraut, R. E., Rice, R., Cool, C. and Fish, R. (1998) Varieties of social influence: The role of utility and norms in the success of a communication medium, Organizational Science, 9, 4, 437-453.

30. Kiss, C. and Bichler, M. (2008) Identification of influencers - Measuring influence in customer networks, Decision Support Systems, 46, 1, 233-253.

31. Koller, T., Goedhart, M. and Wessels, D. (2005) Valuation: Measuring and Managing the Value of Companies, John Wiley, New Jersey.

32. Korte, C. and Milgram, S. (1970) Acquaintance Networks Between Racial Groups: Application of the Small World Method, Journal of Personality and Social Psychology, 15, 2, 101-108.

33. Lambrecht, M. (2008) Sorrell warnt vor Internetblase, Financial Times Deutschland, 2008-02-01.

34. Leskovec, J. and Horvitz, E. (2008) Worldwide buzz: Planetary-scale views on a large instant-messaging network, in Proceeding of the 17th international conference on World Wide Web, April 21-25, Beijing, China, 915-924.

35. Milgram, S. (1967) The small world problem, Psychology Today, 2, 1, 60-67.

36. Morris, M. and Ogan, C. (1996) The Internet as Mass Medium, Journal of Computer-Mediated Communication, 1, 4, http://jcmc.indiana.edu/vol1/issue4/morris.html, 2009-02-12.

37. Rice, R. E. and Aydin, C. (1991) Attitudes Towards New Organizational Technology: Network Proximity as a Mechanism for Social Information Processing, Administrative Science Quarterly, 36, 219-244. 
38. Rogers, E. M. (2003). Diffusion of innovations, Free Press, New York.

39. Shapiro, C. and Varian, H. R. (1998) Information rules - a strategic guide to the network economy, Harvard Business School Press, Boston.

40. Sparrowe, R. T., Liden, R. C., Wayne, S. J. and Kraimer, M. L. (2001) Social Networks and the Performance of Individuals and Groups, Academy of Management Journal, 44, 2, 316-325.

41. Wasserman, S. and Faust, K. (1994) Social Network Analysis: Methods and Applications, Cambridge University Press, Cambridge.

42. Watts, D. J. (2007) The Accidental Influentials, Harvard Business Review, 85, 2, 22-23.

43. Watts, D. J. and Strogatz, S. H. (1998) Collective dynamics of 'small-world' networks, Nature, 393:440-442.

44. Williamson, D. A. (2007) Social Network Marketing: Ad Spending and Usage, http://www.emarketer.com/Report.aspx?code=emarketer_2000478, 2009-02-12.

45. XING (2006) Annual Report for the Shortened Financial Year 2006 of the XING AG.

46. Xu, Y. C., Zhang, C., Xue, L. and Yeo, L. L. (2008) Product adoption in online social network, in Proceedings of International Conference on Information Systems, December 14-17, Paris, France. 